\title{
NEW INEQUALITIES AND INFINITE PRODUCT FORMULAS FOR TRIGONOMETRIC AND THE LEMNISCATE FUNCTIONS
}

\author{
RYO NISHIMURA
}

Abstract. In this paper, we give a new approach to prove inequalities for the Schwab-Borchardt mean, the lemniscatic mean and the arithmetic geometric mean. Additionally, we apply these means to inequalities for trigonometric functions or the lemniscate functions by considering several functional inequalities. One of these applications includes infinite product formulas for the lemniscate function and the arithmetic geometric mean by considering several functional equations.

Mathematics subject classification (2010): 26D05, 26D07, 33B10.

Keywords and phrases: Bivariate means, inequalities, infinite products.

\section{REFERENCES}

[1] J. M. Borwein And P. B. Borerin, Pi and the AGM - A Study in Analytic Number Theory and Computational Complexity, Wiley, New York, 1987.

[2] B. C. CARLSON, Algorithms Invorving Arithmetic and Geometric Means, Amer. Math. Monthly. 78 (1971), 496-505.

[3] A. Levin, A New Class of Infinite Products Generalizing Viéte's Product Formula for $\pi$, Ramanujan J. 10 (2005), 305-324.

[4] A. Levin, A Geometric Interpretation of an Infinite Product for the Lemniscate Constant, Amer. Math. Monthly. 113 (2006), 510-520.

[5] D. S. Mitrinovic, Analytic Inequalities, Springer-Verlag, Berlin, 1970.

[6] E. Neuman, On Gauss Lemniscate Functions and Lemniscatic Mean, Math. Pannonica. 18 (2007), 253-266.

[7] E. Neuman, Two-Sided Inequalities for the Lemniscate Functions, J. Inequal. Spec. Funct. 1 (2007), $1-7$.

[8] E. NEUMAN AND J. SANDOR, On some inequalities involving trigonometric and hyperbolic functions with emphasis on the Cusa-Huygens, Wilker and Huygens inequalities, Math. Inequal. Appl. 13 (4) (2010), 715-723.

[9] E. Neuman And J. SAndor, On the Schwab Borchardt mean, Math. Pannonica. 14 (2) (2003), 253-266. 